\title{
2-S23-1 Symposium23
}

\section{The role of central nervous system in the regulation of glucose homeostasis}

\section{Hiroko Ikeda}

Dept. Pathophysiol. Ther., Hoshi Univ. Sch. Pharm. Pharmaceut. Sci.

Since the disturbance of glucose metabolism causes diabetes mellitus and related diseases, it is important to regulate plasma glucose levels properly. Plasma glucose levels are regulated by the balance with glucose production and glucose utilization. It is well known that glucagon and insulin secreted by the pancreas play important roles in the regulation of plasma glucose levels. In addition, the liver regulates blood glucose levels by glycogenolysis and gluconeogenesis. These functions are controlled by autonomic nerves. In contrast, recent evidence suggested that the central nervous system (CNS) regulates blood glucose levels. It is known that the hypothalamus regulates energy homeostasis including glucose metabolism. Moreover, it is reported that the hypothalamus regulates the sympathetic and parasympathetic nerves. We have shown that an anti-psychotic drug olanzapine increases plasma glucose levels through sympathetic nerves. Thus, it is suggested that the CNS regulates plasma glucose levels through autonomic nerves. We have recently focused on central dopaminergic functions, which is one of the action sites of olanzapine, and found how central dopaminergic functions regulate blood glucose levels. In this symposium, I would like to review how the CNS regulates glucose metabolism and to introduce our recent research. 